\title{
Self-predictions overweight strength of current intentions
}

\author{
Derek J. Koehler *, Connie S.K. Poon ${ }^{1}$ \\ Department of Psychology, University of Waterloo, Waterloo, Ont., Canada N2L $3 G 1$ \\ Received 1 February 2005; revised 20 July 2005 \\ Available online 23 September 2005
}

\begin{abstract}
We suggest that people's predictions of their future behavior overweight the strength of their current intentions, and underweight situational or contextual factors that influence the ease with which intentions are translated into action. As expected by this account, we find that self-predictions closely follow ratings of current intention strength, and that the actual probability of the behavior being predicted does not increase with intention strength to the extent implied by self-predictions (Study 1). We also find that manipulations designed to strengthen intentions to carry out a behavior have a larger impact on self-predictions than on the behavior being predicted (Studies 1 and 2), whereas a manipulation designed to influence the ease with which intentions are translated into behavior has a larger impact on actual behavior than on self-predictions (Study 2). Observers' predictions of another's behavior do not follow the same pattern (Study 3).

(C) 2005 Elsevier Inc. All rights reserved.
\end{abstract}

Keywords: Prediction of future behavior; Intention; Optimistic biases

Many decisions we face at any given time require us to predict our actions the next day, month, or year. A patient diagnosed with Type-2 diabetes may have to choose between taking a new medication (with possible side effects) and managing the condition through diet and exercise alone. An important consideration in this decision is whether the patient anticipates being able to adhere to the prescribed diet and exercise regimen. A consumer choosing between two credit cards, one with a high interest rate but no annual fee, and the other with a lower interest rate but a substantial annual fee, must evaluate the likelihood that interest charges will be avoided by paying off the balance in full each month. In decisions such as these, inaccurate self-predictions of future behavior can be costly, leading to choices that are later regretted.

Surprisingly, despite their importance in making good decisions, self-predictions are often inaccurate (e.g., Osberg \& Shrauger, 1986; Vallone, Griffin, Lin, \& Ross, 1990),

\footnotetext{
* Corresponding author. Fax: +1 5197468631.

E-mail address: dkoehler@uwaterloo.ca (D.J. Koehler).

${ }^{1}$ Present address: Department of Psychology at the University of Hong Kong.
}

even for behaviors largely under the individual's control (e.g., Epley \& Dunning, 2000). Notably, self-predictions are often overly optimistic (e.g., Armor \& Taylor, 1998; Buehler, Griffin, \& Ross, 1994; Kahneman \& Lovallo, 1993; Taylor \& Brown, 1988; Weinstein, 1980). The present research offers a characterization of the self-prediction process that helps to identify the conditions under which selfpredictions are expected to be more or less optimistically biased.

\section{Intention-based prediction}

We suggest that when assessing how likely they are to carry out some future behavior, people begin with an evaluation of their current intentions with regard to that behavior. In the present analysis, intentions are assumed to be tied to specific behaviors, and to vary in strength, that is, the extent to which the individual feels committed (at a particular moment) to carrying out that behavior. In this paper, we will argue that although current intention strength can be a useful cue in predicting the likelihood of future behavior, people typically place too much weight on this cue when making self-predictions. 
The usefulness of current intention strength as a predictor of future behavior has been demonstrated in an extensive program of research based on the theory of planned behavior (e.g., Ajzen, 1991; Ajzen \& Madden, 1986). Generally, the stronger the current intention, the more likely it is that the behavior will be carried out in the future. In this research, however, current intentions typically account for about $20-40 \%$ of variance in later behavior (Ajzen, 1991; Gollwitzer, 1999; Ouellette \& Wood, 1998; Sutton, 1998). In other words, the predictive validity of current intentions is generally far from perfect; a substantial amount of variance in behavior remains unexplained by intentions. As we all know, certain behaviors may not occur despite our best intentions.

What factors influence the predictive validity of current intentions? The validity with which current intention strength (evaluated at $T_{1}$, the time of prediction) can be used to predict later behavior (at $T_{2}$, the time at which the opportunity arises to carry out the target behavior) may be separated into two conceptual components: (1) the temporal stability of intention strength over the period between $T_{1}$ and $T_{2}$; and (2) the translatability of intentions at $T_{2}$ in terms of how readily they produce action. Intention strength at $T_{1}$ will be a valid predictor of behavior at $T_{2}$ only to the extent that intention strength is stable over the period between $T_{1}$ and $T_{2}$, and intention strength at $T_{2}$ is readily translated into action.

The translatability of intentions can be influenced by various situational or contextual factors, which may or may not be considered by the individual at the time of prediction. These influences fall into a category that Lewin (1951) described as "channel" factors (see Ross \& Nisbett, 1991), a classic example being the provision of a map to students following a persuasive appeal to participate in a tetanus inoculation program (Leventhal, Singer, \& Jones, 1965). Although all the students exposed to the persuasive appeal generally reported strong intentions to participate, those provided with a campus map indicating the location of the health center were much more likely to go for their inoculation than were those not given a map. In terms of our analysis, the persuasive appeal can be viewed as strengthening students' intentions to participate in the inoculation program, while the provision of a map can be viewed as influencing the readiness with which those strengthened intentions are translated into behavior.

To make the best use of current intentions as a cue in self-predictions, one must take into account the predictive validity of this cue, among other considerations. Basic statistical principles dictate that, to maximize predictive accuracy, the lower the validity of current intention strength as a predictor of later behavior, the less weight one should place on it in the prediction process (i.e., the more regressive the prediction should be with respect to current intention strength). Previous research, however, indicates that intuitive predictions are often insensitive to the validity of predictive cues. Kahneman and Tversky (1973) observed that people engage in "prediction by evaluation," in which predictions (e.g., of a teacher's competence five years after leaving teachers college) are just as extreme as the evaluations (e.g., of the teacher's performance in a practice lesson during college) upon which they are based. Because they are largely insensitive to the validity of the predictor variable, intuitive predictions are non-regressive and hence too extreme. In further developing this analysis, Griffin and Tversky (1992) suggested that confidence in judgment is based primarily on the strength of the impression conveyed by the available evidence, with little regard given to the "weight" (or validity) of that evidence. From this view, if self-predictions are based primarily on strength of intention, which reflects one's goals and aspirations, they should be most overly optimistic when intention strength is high but its predictive validity is low.

We propose that an evaluation of current intention strength serves as a natural starting point in the self-prediction process. Using the terminology of Kahneman and Frederick (2002), we suggest that people use current intention strength as a readily available, easily evaluated "heuristic attribute" in intuitive self-prediction. This heuristic attribute tends to be substituted for the more difficult judgment of the "target attribute," that is, the probability of the target behavior. Adjustments may then be made in light of additional factors that influence future behavior to the extent that they are recognized as relevant, but such adjustments are likely to be insufficient (Epley \& Gilovich, 2001; Tversky \& Kahneman, 1974). Consequently, we expect that current intention strength will be overweighted relative to its impact on the future behavior being predicted, and predictive factors unrelated to current intention strength will be underweighted.

Our analysis draws on some important insights by Ross and colleagues in their work on intuitive social prediction (e.g., Dunning, Griffin, Milojkovic, \& Ross, 1990; Griffin, Dunning, \& Ross, 1990; Vallone et al., 1990). They observe that people tend to be overconfident in predicting both their own and others' future behavior, and attribute such overconfidence to a tendency to explain behavior on the basis of inferred internal dispositions (i.e., lay dispositionism, see, e.g., Dunning et al., 1990) and an accompanying failure to accommodate uncertainty about future situational circumstances that often prove to have a dramatic impact on behavior (Griffin et al., 1990; Vallone et al., 1990). Our analysis, by contrast, focuses on experienced intentions rather than inferred dispositions, and we provide direct empirical evidence for the role of current intentions in self-predictions. In our analysis, people's insufficient allowance for the predictive uncertainty arising from situational factors is treated as a consequence of the focus on current intentions at the time of self-prediction. Our framework can help identify the conditions under which self-predictions are likely to be more or less overly optimistic, a question that is not directly addressed in the research by Ross and colleagues. The broader insight offered in their work, however, that intuitive behavioral predictions focus on internal factors and often fail to recognize the powerful 
impact of situational factors on behavior, is a central component of our analysis.

\section{Overview of present studies}

We present results of three studies supporting our depiction of the self-prediction process. Study 1 reveals a close correspondence between current intentions and predictions of future behavior. A manipulation that influences current intentions, furthermore, is shown to have a more pronounced effect on self-predictions than on the target behavior. Study 2 demonstrates that self-predictions are simultaneously overly sensitive to a manipulation designed to influence current intention strength and insufficiently sensitive to a manipulation unrelated to current intentions, relative to the impact of such manipulations on the target behavior. Study 3 indicates that predictions by observers do not exhibit the same pattern of biases as self-predictions. Together, these studies are the first to establish that self-predictions overweight current intentions, with predictable consequences for their accuracy.

\section{Study 1}

We examined predicted and actual participation in a blood donation clinic. Blood donation is a particularly interesting target behavior from the present perspective, as many people express good intentions but relatively few actually donate blood. According to Canadian Blood Services (2002), for example, $75 \%$ of surveyed Canadians reported that they "have plans to donate or might donate in the future," but relatively few eligible Canadians actually donate blood.

\section{Method}

A week-long blood donation clinic is held once per academic term at the University of Waterloo's Student Life Centre. One to three weeks in advance of the clinic, students eligible to donate blood $(N=230)$ who were studying or eating at the Student Life Centre were asked to complete a short questionnaire regarding attitudes toward blood donation.

The questionnaire consisted of two one-page sections. One section informed respondents of the upcoming blood donation clinic, and asked them to estimate their probability of donating blood at the clinic on a $0-100 \%$ scale with $10 \%$ increments. Respondents also rated their agreement with the statement "Right now, as I think about it, I strongly intend to donate blood at the July 14-22 blood donor clinic" on a 1 ("strongly disagree") to 9 ("strongly agree") scale, which we use as a measure of current intention strength. The order of the prediction and intention measures was counterbalanced across respondents.

The other section consisted of a set of positive and largely unobjectionable statements regarding blood donation (e.g., "It is well worth taking the time to donate blood";
"More Canadians should donate blood"; "A blood shortage in Canada would be unacceptable"). Respondents rated their agreement with each statement on a 9-point scale. We expected that completing these ratings would temporarily activate or enhance intentions to donate blood. Participants were randomly assigned to complete the agreement items either before (agreement first condition) or after (prediction first condition) making their predictions.

Respondents were asked whether we could re-contact them by e-mail to complete a brief follow-up questionnaire. Those who completed the follow-up questionnaire were entered in a draw to win a $\$ 50$ gift certificate from a local restaurant. The follow-up questionnaire, sent the day after the blood donation clinic had ended, asked respondents whether or not they had donated blood at the clinic.

\section{Results}

Of the 230 respondents who were eligible to donate blood, $218(95 \%)$ were willing to be re-contacted for the follow-up questionnaire. Of those, $137(63 \%)$ responded to the follow-up questionnaire. Those who responded did not differ from those who did not with regard to their predictions, nor did this factor interact with the order manipulation (agreement first vs. prediction first) in its influence on predictions, and the order manipulation itself did not affect the likelihood of responding; the same was true for those who were willing to be re-contacted vs. those who were not. One respondent failed to complete the intention rating; our analyses are restricted to the remaining 136 respondents. Table 1 shows the mean predicted and actual target behavior probability by condition for each of the studies reported here.

As expected, respondents expressed stronger intentions to donate blood after completing the agreement items $(M=5.8 ; n=70)$ than they did before completing the agreement items $(M=3.8 ; n=66), F(1,134)=17.0, M S E=8.0$, $p<.001$. Furthermore, participants gave higher probability estimates after completing the agreement items $(M=54 \%)$ than they did before completing the agreement items $(M=31 \%), F(1,134)=15.4, M S E=1163, p<.001$.

To ascertain that the influence of the order manipulation on participants' probability judgments was mediated by its effect on current intention strength, we conducted a series of regression analyses (cf. Baron \& Kenny, 1986). First, we regressed participants' probability judgments on the order manipulation (which was coded as agreement first or prediction first) and obtained a significant effect, $\beta=.32, t(134)=3.92, p<.001$. Second, we regressed participants' intention strength ratings on the order manipulation, $\beta=.34, t(134)=4.13, p<.001$. Third, we regressed participants' probability judgments on both the order manipulation and their intention strength ratings. In this regression, intention strength ratings had a significant effect on probability judgments, $\beta=.90, t(133)=22.77, p<.001$, whereas the effect of the order manipulation on probability judgments was no longer significant, $\beta=.02, t(133)=.50$, 
Table 1

Mean predicted and actual probability of target behavior (in percent) in Studies 1-3, by experimental condition

\begin{tabular}{|c|c|c|c|c|c|c|}
\hline & \multirow{2}{*}{\multicolumn{2}{|c|}{$\frac{\text { Study } 1}{\text { First task }}$}} & \multicolumn{4}{|c|}{ Studies 2 and 3} \\
\hline & & & \multicolumn{2}{|c|}{ Importance } & \multicolumn{2}{|c|}{ Reminder } \\
\hline & Prediction & Agreement & Low & High & No & Yes \\
\hline Self-prediction & 31 & 54 & 68 & 81 & 74 & 76 \\
\hline Behavior & 17 & 23 & 17 & 22 & 13 & 26 \\
\hline Observer prediction & - & - & 50 & 52 & 47 & 55 \\
\hline
\end{tabular}

$p=.62$. A Goodman test indicated that the standardized regression co-efficient $(\beta)$ for the order manipulation in the third regression equation decreased significantly relative to that in the first regression equation $(z=4.06$, $p<.001)$. Results of this analysis are consistent with our expectation that the influence of the order manipulation on self-predictions was mediated by its influence on current intention strength.

Predictions were generally too optimistic, with the predicted probability of donating blood at the clinic ( $M=43 \%$ ) exceeding the actual rate of donation (27 out of the 136 respondents, or $20 \%$, reported having donated blood at the clinic). Fig. 1 indicates that the probability of donating blood did not increase as steeply with intention strength as was implied by the self-predictions. Although those who completed the agreement items before making predictions gave a notably higher probability of donating blood, their actual rate of donation (16 out of 70 , or $23 \%$ ) was only slightly - and not statistically significantly-greater than that of the respondents who made predic-

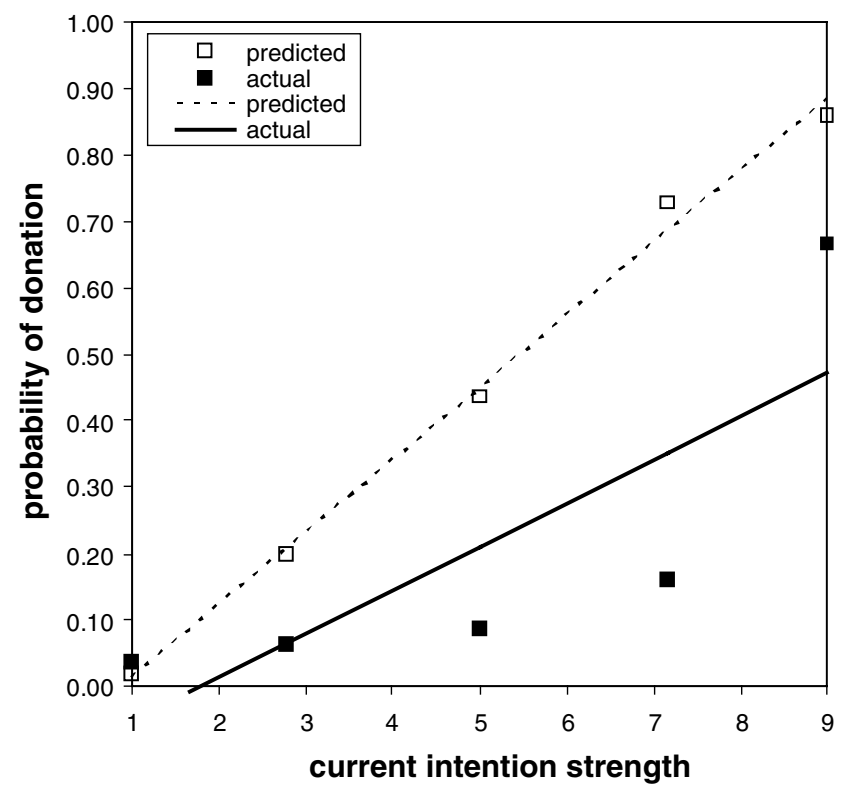

Fig. 1. Mean predicted and actual probability of blood donation in Study 1 as a function of intention strength at the time of prediction. Note: Mean predicted and actual probabilities of blood donation were calculated from data aggregated over intention strength ratings of 2-4 and of 6-8 so that each point in the figure represents an approximately equal number of observations ( $n=29,32,23,25,27$, moving from low to high intention strength). Weighted least squares regression was then used to fit the resulting data points. tions before completing the agreement items (11 out of 66, or $17 \%), \chi^{2}(1, N=136)=.82$. In other words, as expected, the order manipulation designed to influence current intentions had a more pronounced influence on self-predictions than on actual behavior.

\section{Study 2}

In Study 2, we introduce another manipulation designed to influence intentions at the time of prediction; furthermore, we include a manipulation designed to influence the readiness with which intentions can be translated into action (i.e., translatability of intentions). According to the present account, the former should have a more pronounced impact on self-predictions than on actual behavior (replicating the results of Study 1), while the latter-because it was not expected to influence intentions - should have a less pronounced impact on self-predictions than on behavior.

\section{Method}

University of Waterloo undergraduates $(N=170)$, who had just completed an unrelated experiment for extra credit in their introductory psychology course, were given a recruitment form seeking volunteers for a brief, web-based study that would be operational for a one-week period beginning several weeks in the future. They were not asked to make an immediate commitment, but were told that it would be useful to the graduate student who was conducting the study to have a realistic estimate of how many participants she could expect. For this purpose, respondents were asked to estimate the probability that they would participate in the web study on a scale running from $0 \%$ (certain that you will not participate) to $100 \%$ (certain that you will participate) in increments of $5 \%$. All respondents were asked to provide their e-mail address so that, regardless of whether they chose to participate, they could be sent an informational message at the end of the term explaining the purpose and results of the study. To maintain the plausibility of our cover story, no further measures (e.g., intention strength ratings) were elicited from participants. The bottom part of the recruitment form, which was to be removed and taken home by the respondent, indicated the website address for the study and the dates it would be running, and provided an ID number with which to log on to the web study. 
Some of the respondents were informed that their participation in the web study was crucial for the graduate student conducting the study to be able to receive her degree, as she was having difficulty finding a sufficient number of participants. Others were told that their participation would be helpful but not absolutely crucial as the graduate student had already recruited a sufficient number of participants. We assumed that the former cover story (high importance condition) would activate stronger intentions to complete the web study than would the latter (low importance condition). We also manipulated the ease with which intentions to participate in the study could be translated into action by promising (and actually sending) a reminder e-mail message to one group of respondents (reminded condition) on the first day of the one-week period in which the web study was scheduled to be operational. For the remaining respondents (unreminded condition), no such reminder was promised (or sent).

The web study was activated and the e-mail reminders sent (to the reminded group only) early on the first day of the designated one-week period. The reminder indicated the dates during which the study would be running and the website address. ${ }^{2}$ The ID number and time and date of participation was logged for each participant who visited the website.

\section{Results}

We excluded from analysis the data from five respondents who failed to provide a probability estimate, six who did not detach and retain the bottom portion of the recruitment form, and two more in the reminder condition who failed to provide an e-mail address. Because the next study, reported below, used a design in which an observer was yoked to each respondent in the current study, for consistency we also excluded data from three respondents for whom the yoked observers failed to provide probability judgments, leaving 154 respondents in the final sample.

Overall, self-predictions were much too optimistic: the mean judged probability of participating in the web study was $75 \%$, but only 30 of the 154 respondents (19\%) actually participated several weeks later. As the two experimental manipulations did not exert any interacting influence on predicted or actual behavior, we report only their main effects (see Table 1).

The importance manipulation significantly influenced self-predictions $(M=81 \%$ for high importance vs. $M=68 \%$ for low importance condition), $F(1,150)=13.30$, $M S E=485, p<.01$. By comparison, the difference in actual participation in the web study between the two importance

\footnotetext{
${ }^{2}$ In fact, the study was run over the course of two academic terms, with one minor procedural variation. In the initial version, the reminder message did not include respondents' assigned ID number (nor were they promised that it would); in the subsequent version, it did include this information (and respondents were told that it would). This factor had no effect on the results and so is disregarded in our analyses.
}

conditions was quite small, with only a weak and statistically non-significant tendency for the high importance group to participate at a higher rate (17 of 76 respondents, or $22 \%$ ) than the low importance group (13 of 78 respondents, or $17 \%), \chi^{2}(1, N=154)=0.80$.

The reminder manipulation, by contrast, had only a small, statistically non-significant effect on self-predictions ( $M=76 \%$ for reminded vs. $M=74 \%$ for the unreminded condition), $F(1,150)<1$. In terms of actual participation, however, students sent a reminder were noticeably more likely to participate in the web study ( 20 of 78 respondents, or $26 \%$, completed the web study) than were those not sent a reminder (10 of 76 respondents, or $13 \%$ ), $\chi^{2}(1, N=154)=3.89, p<.05$.

As expected, then, the importance manipulation designed to influence current intention strength exerted a larger effect on self-predictions (mean probability difference $=13 \%$ ) than it did on actual behavior (mean difference $=5 \%$ ). By contrast, the reminder manipulation designed to influence the ease with which intentions are translated into behavior exerted a smaller effect on self-predictions (mean difference $=2 \%$ ) than it did on actual behavior (mean difference $=13 \%$ ).

Taken together, the results of Studies 1 and 2 clearly support our contention that self-predictions overweight the strength of current intentions. Participants in both studies appeared to have made insufficient allowance for the imperfect validity of current intentions as a predictor of later behavior. As noted in the Introduction, the predictive validity of current intentions is affected by factors influencing the temporal stability and the translatability of intentions. The reminder manipulation may well have influenced the temporal stability of intention strength (being reminded may have "reactivated" good intentions) as well as its translatability into action once the opportunity to act was available (being reminded may have prompted behavior in line with existing intentions). Either way, the finding that the reminder manipulation had a greater impact on behavior than was implied by the self-predictions supports our general claim that factors influencing the predictive validity of current intentions are underweighted in the self-prediction process.

\section{Study 3}

Because we have direct, privileged access to our own current intentions, but not to those of others, an evaluation of current intentions is less likely to play a central role when we predict the behavior of another person than when we predict our own behavior. Relative to self-predictions, then, predictions of others' behavior may have greater latitude to take predictive considerations other than current intentions into account. This possibility is tested in Study 3.

\section{Method}

An "observer" was yoked to each of the 154 respondents whose data were analyzed in Study 2. The observers, 
like the "actors" of the previous study, were undergraduates at the University of Waterloo enrolled in an introductory psychology course. Each observer was provided with an identical copy of the recruitment form that had been presented to the actor, and was informed of the date on which the actor had completed it. The observer was asked to estimate the probability that the actor had participated in the web study, using the same scale as that used by the actors in making their self-predictions. The observer did not see the actor's self-prediction.

\section{Results}

Observers were generally less optimistic in their predictions than were the actors, assigning a mean probability of participating in the web study of $51 \%$ (vs. $75 \%$ for the actor's self-predictions), consistent with previous findings (e.g., Buehler et al., 1994; Epley \& Dunning, 2000; MacDonald \& Ross, 1999). This result is also consistent with the present account on the assumption that prosocial behaviors of the kind investigated here are associated with good intentions, which are expected to be strongly associated with self-predictions but not observers' predictions (cf. Kruger \& Gilovich, 2004).

Observers' predictions were largely insensitive to the importance manipulation $(M=52 \%$ for high importance vs. $M=50 \%$ for low importance condition), $F(1,150)<1$, $M S E=660$. The difference between the high and low importance conditions in the observers' predictions $(2 \%)$ is considerably closer to that observed in actual behavior $(5 \%)$ than was found in the actors' predictions (13\%) (see Table 1). This finding is consistent with our expectation that observers' predictions would be less sensitive than self-predictions to factors designed to influence intentions.

Also in contrast to the self-predictions, observers' predictions were influenced by the reminder manipulation ( $M=55 \%$ for reminded vs. $M=47 \%$ for the unreminded condition), $F(1,150)=3.82, p=.052$. The difference between the reminded and unreminded conditions in the observers' predictions $(8 \%)$ is considerably closer to that observed in actual behavior (13\%) than was found in the actor's predictions (2\%) (see Table 1). This finding is consistent with our expectation that the observers' predictions would be more sensitive to predictive considerations unrelated to intentions than would the self-predictions. The tendency to focus on current intentions when making self-predictions can lead people to underestimate the impact of other factors on their own behavior even when they recognize the impact of those factors on the behavior of others.

\section{General discussion}

Our research is the first to provide direct evidence that self-predictions overweight current intentions: people tend to be overly certain that the likelihood of their carrying out some future behavior coincides with the strength of their current intentions. Intuitive self-predictions make insufficient allowance for the imperfect predictive validity of current intentions or the role of other factors that also influence future behavior.

The current findings fit nicely with prior research showing that people's self-predictions of when they will complete tasks are routinely more optimistic than can be justified by their actual completion times (for a review, see Buehler, Griffin, \& Ross, 2002). This planning fallacy has been linked to the construction of a best-case scenario or a plan in which events or steps from the commencement to the completion of the project proceed as well as they possibly can (e.g., Buehler et al., 1994). It is worth noting that our explanatory approach is distinct from that offered in the planning fallacy literature. Instead of focusing on people's construction of scenarios, we attribute self-prediction bias to an overweighting of current intentions experienced by the actor (i.e., how strongly the actor feels committed to carry out a particular behavior) at the time of prediction. Our explanatory approach may help illuminate a puzzling finding in the planning fallacy literature: prompting people to generate pessimistic scenarios in which things do not go as smoothly as planned has surprisingly little impact in reducing the magnitude of the planning fallacy (Newby-Clark, Ross, Buehler, Koehler, \& Griffin, 2000). One might expect that if optimistic predictions result from the construction of a scenario by which everything goes smoothly, drawing attention to a pessimistic scenario would be a useful de-biasing technique. If self-predictions are based on current intentions, however, as we have suggested, we would not necessarily expect pessimistic scenarios to have a de-biasing effect, as construction of such scenarios may neither mitigate people's intentions to carry out the target behavior, nor reduce the weight they place on current intentions in making self-predictions.

Loewenstein and colleagues (e.g., Loewenstein, 1996) have observed another form of bias in self-predictions: people tend to underestimate the impact on their future preferences of being in a "hot" visceral state (e.g., hungry, sexually aroused) when they are in a "cold" state at the time of prediction, and vice versa. As applied to our account, according to which self-predictions are based on an evaluation of current intention strength, Loewenstein's research could be taken to imply that self-predictions made when "in the grip" of a strong intention will fail to anticipate the impact of changes in those intentions over time. There is an important conceptual difference, however, between the two lines of research. Loewenstein and colleagues' research focuses on intense visceral states that are - almost by definition - short-lived, and explores how changes in experienced visceral states can produce corresponding changes in preference that people may not correctly anticipate. By contrast, our theoretical framework suggests that even temporally stable intentions will be overweighted in self-predictions, as the evaluation of intention strength fails to capture other important determinants of behavior. 
Also relevant to the study of self-predictions is research by Liberman and Trope (1998; Trope \& Liberman, 2003) on the construal of future events. They argue that people tend to adopt high-level construals (e.g., focus on desirability considerations) for temporally distant events, and lower-level construals (e.g., focus on feasibility considerations) for proximal events. Liberman and Trope's construal level theory has provided new insights into the determinants of intentions regarding behaviors of varying temporal distance (see, e.g., Eyal, Trope, Liberman, \& Walther, 2004). Our research complements theirs by specifying the role of intention strength in the self-prediction process. In doing so, our work provides a basis for understanding differences between predictions of one's own behavior vs. that of others, on the grounds that we have direct, privileged access to our own current intentions but not to those of others. Differences between self- and other-predictions, such those shown in Study 3, would not necessarily be predicted from construal level theory alone (e.g., Nussbaum, Trope, \& Liberman, 2003). Furthermore, by placing current intentions at the heart of the self-prediction process, our theoretical account can, taken in conjunction with construal level theory, be used to predict the degree of optimism in self-predictions regarding behaviors of varying temporal distance. Specifically, to the extent that current intention strength regarding more temporally distant behavior is determined more by desirability considerations and less by feasibility considerations (cf. Eyal et al., 2004), self-predictions based on current intentions would be expected to become increasingly optimistic as the target behavior becomes more distant in the future.

\section{Acknowledgments}

This research was supported by a grant to the first author from the Social Sciences and Humanities Research Council of Canada. We thank Eva Adam, Scott McAllister, and Catherine Rawn for their assistance with the studies. This research was presented in seminars at the University of Chicago, Duke University, Stanford University, UCLA, and the University of Waterloo. We are grateful for the helpful comments and suggestions offered by participants in these seminars.

\section{References}

Ajzen, I. (1991). The theory of planned behavior. Organizational Behavior and Human Decision Processes, 50, 179-211.

Ajzen, I., \& Madden, T. J. (1986). Prediction of goal-directed behavior: Attitudes, intentions, and perceived behavioral control. Journal of Experimental Social Psychology, 22, 453-474.

Armor, D. A., \& Taylor, S. E. (1998). Situated optimism: Specific outcome expectancies and self-regulation. In M. P. Zanna (Ed.). Advances in experimental social psychology (Vol. 30, pp. 309-379). New York: Academic Press.

Baron, R. M., \& Kenny, D. A. (1986). The moderator-mediator variable distinction in social psychological research: Conceptual, strategic and statistical considerations. Journal of Personality and Social Psychology, $51,1173-1182$.

Buehler, R., Griffin, D., \& Ross, M. (1994). Exploring the "planning fallacy": Why people underestimate their task completion times. Journal of Personality and Social Psychology, 67, 366-381.

Buehler, R., Griffin, D., \& Ross, M. (2002). Inside the planning fallacy: The causes and consequences of optimistic time predictions. In T. Gilovich, D. Griffin, \& D. Kahneman (Eds.), Heuristics and biases: The psychology of intuitive judgment (pp. 250-270). Cambridge: Cambridge University Press.

Canadian Blood Services (2002). Not enough Canadians rolling up their sleeves. Press release.

Dunning, D., Griffin, D., Milojkovic, J. D., \& Ross, L. (1990). The overconfidence effect in social prediction. Journal of Personality and Social Psychology, 58, 568-581.

Epley, N., \& Dunning, D. (2000). Feeling "holier than thou": Are selfserving assessments produced by errors in self- or social prediction? Journal of Personality and Social Psychology, 79, 861-875.

Epley, N., \& Gilovich, T. (2001). Putting adjustment back in the anchoring-and-adjustment heuristic: Self-generated versus experimenter-provided anchors. Psychological Science, 12, 391-396.

Eyal, T., Trope, Y., Liberman, N., \& Walther, E. (2004). The pros and cons of temporally near and distant action. Journal of Personality and Social Psychology, 86, 781-795.

Gollwitzer, P. M. (1999). Implementation intentions: Strong effects of simple plans. American Psychologist, 54, 493-503.

Griffin, D. W., Dunning, D., \& Ross, L. (1990). The role of construal process in overconfident predictions about the self and others. Journal of Personality and Social Psychology, 59, 1128-1139.

Griffin, D., \& Tversky, A. (1992). The weighing of evidence and the determinants of confidence. Cognitive Psychology, 24, 411-435.

Kahneman, D., \& Frederick, S. (2002). Representativeness revisited: Attribute substitution in intuitive judgment. In T. Gilovich, D. Griffin, \& D. Kahneman (Eds.), Heuristics and biases: The psychology of intuitive judgment (pp. 49-81). Cambridge: Cambridge University Press.

Kahneman, D., \& Lovallo, D. (1993). Timid choices and bold forecasts. A cognitive perspective on risk taking. Management Science, 39, 17-31.

Kahneman, D., \& Tversky, A. (1973). On the psychology of prediction. Psychological Review, 80, 237-251.

Kruger, J., \& Gilovich, T. (2004). Actions, intentions, and self assessment: The road to self-enhancement is paved with good intentions. Personality and Social Psychology Bulletin, 30, 328-339.

Leventhal, H., Singer, R. P., \& Jones, S. H. (1965). The effects of fear and specificity of recommendation. Journal of Personality and Social Psychology, 2, 20-29.

Lewin, K. (1951). Field theory in social science. New York: Harper and Row.

Liberman, N., \& Trope, Y. (1998). The role of feasibility and desirability considerations in near and distant future decisions: A test of temporal construal theory. Journal of Personality and Social Psychology, 75, $5-18$.

Loewenstein, G. (1996). Out of control: Visceral influences on behavior. Organizational Behavior and Human Decision Processes, 65, 272-292.

MacDonald, T. K., \& Ross, M. (1999). Assessing the accuracy of predictions about dating relationships: How and why do lovers' predictions differ from those made by observers? Personality and Social Psychology Bulletin, 25, 1417-1429.

Newby-Clark, I. R., Ross, M., Buehler, R., Koehler, D. J., \& Griffin, D. (2000). People focus on optimistic and ignore pessimistic scenarios while predicting their task completion times. Journal of Experimental Psychology: Applied, 6, 171-182.

Nussbaum, S., Trope, Y., \& Liberman, N. (2003). Creeping dispositionism: The temporal dynamics of behavior prediction. Journal of Personality and Social Psychology, 84, 485-497.

Osberg, T. M., \& Shrauger, J. S. (1986). Self-prediction: Exploring the parameters of accuracy. Journal of Personality and Social Psychology, $51,1044-1057$. 
Ouellette, J. A., \& Wood, W. (1998). Habit and intention in everyday life: The multiple processes by which past behavior predicts future behavior. Psychological Bulletin, 124, 54-74.

Ross, L., \& Nisbett, R. E. (1991). The person and the situation. New York: McGraw-Hill.

Sutton, S. (1998). Predicting and explaining intentions and behavior: How well are we doing? Journal of Applied Social Psychology, 28, 1317-1338.

Taylor, S. E., \& Brown, J. D. (1988). Illusion and well-being: A social psychological perspective on mental health. Psychological Bulletin, 103, 193-210.
Trope, Y., \& Liberman, N. (2003). Temporal construal. Psychological Review, 110, 403-421.

Tversky, A., \& Kahneman, D. (1974). Judgment under uncertainty: Heuristics and biases. Science, 185, 1124-1131.

Vallone, R. P., Griffin, D. W., Lin, S., \& Ross, L. (1990). Overconfident prediction of future actions and outcomes by self and others. Journal of Personality and Social Psychology, 58, 582-592.

Weinstein, N. D. (1980). Unrealistic optimism about future life events. Journal of Personality and Social Psychology, 39, 806-820. 